OPEN ACCESS

Edited by:

Hans J. Grabe,

University of Greifswald, Germany

Reviewed by:

Lukas Pirpamer,

Medical University of Graz, Austria

Zhen Yuan,

University of Macau, China

*Correspondence:

Yingchun Zhang

yzhang94@uh.edu

Received: 07 August 2019 Accepted: 20 February 2020

Published: 10 March 2020

Citation:

Wu Z, Peng Y, Selvaraj S, Schulz PE and Zhang Y (2020)

Development of Brain Structural Networks Over Age 8: A Preliminary Study Based on Diffusion Weighted

Imaging.

Front. Aging Neurosci. 12:61. doi: 10.3389/fnagi.2020.00061

\section{Development of Brain Structural Networks Over Age 8: A Preliminary Study Based on Diffusion Weighted Imaging}

\author{
Zhanxiong Wu ${ }^{1,2}$, Yun Peng' ${ }^{2}$, Sudhakar Selvaraj ${ }^{3}$, Paul E. Schulz ${ }^{4}$ and Yingchun Zhang ${ }^{2 *}$ \\ ${ }^{1}$ School of Electronic Information, Hangzhou Dianzi University, Hangzhou, China, ${ }^{2}$ Department of Biomedical Engineering, \\ University of Houston, Houston, TX, United States, ${ }^{3}$ Louis A. Faillace, MD, Department of Psychiatry and Behavioral \\ Sciences, The McGovern Medical School of UT Health Houston, Houston, TX, United States, ${ }^{4}$ Department of Neurology, \\ The McGovern Medical School of UT Health Houston, Houston, TX, United States
}

Brain structural network changes provide key information about the aging process of the brain. Unfortunately, there has yet to be a detailed characterization of these structural networks across different age groups. Efforts to classify these networks have also been hampered by their reliance on technically limited traditional methods, which are unable to track multiple fiber orientations within a voxel and consequently are prone to false detection and artifacts. In this study, a newly developed Ensemble Average Propagator (EAP) based probabilistic tractography method was applied to construct a structural network, with the strength of the link between any two brain functional regions estimated according to the alignment of the EAP along connecting pathways. Age-related changes in the topological organization of human brain structural networks were thereby characterized across a broad age range (ages 8-75 years). The data from 48 healthy participants were divided into four age groups (Group 1 aged 8-15 years; Group 2 aged 25-35 years; Group 3 aged 45-55 years; and, Group 4 aged 65-75 years; $N=12$ per group). We found that the brain structural network continues to strengthen during later adolescence and adulthood, through the first 20-30 years of life. Older adults, aged 65-75, had a significantly less optimized topological organization in their structural network, with decreased global efficiency and increased path lengths versus subjects in other groups. This study suggests that probabilistic tractography based on EAP provides a reliable method to construct macroscale structural connectivity networks to capture the age-associated changes of brain structures.

Keywords: magnetic resonance imaging, diffusion weighted imaging, ensemble average propagator, structural network, brain development

\section{INTRODUCTION}

Human brain structural networks are functionally modular and connect effectively through neural bundles to meet the needs for complex cognitive tasks (Schmahmann et al., 2007; Lerch et al., 2017). This neural fiber connectivity enables the communication between the various regions of the brain (Bassett et al., 2011) and its integrity is pivotal for individual health. Due to 
the development of non-invasive imaging technologies, such as diffusion-weighted imaging (DWI), our knowledge of these structural pathways have vastly improved. DWI characterizes structural connectivity networks across brain regions in-vivo by calculating the number of streamlines or the probability of connections (Frank, 2002; Tuch et al., 2002; Anderson, 2005; Maier-Hein et al., 2017). The demonstrated connectivity patterns can then be assessed through graph-based analyses that outline the complex structural substrates of cognition (Betzel et al., 2014). This approach has been effectively employed to identify densely interconnected structural hub regions that are critical to efficient neuronal signaling and communication (van den Heuvel and Sporns, 2013).

Aging has been recognized as a significant factor affecting brain functions. Specifically, a growing body of evidence suggests that brain structure is altered as age increases. Although the fundamental cause of these age-related white matter changes has yet to be fully understood, current theories tie them to changes in the axonal diameter and myelination, synaptic pruning, and modification (Onoda et al., 2012; Dennis and Thompson, 2014; Geerligs et al., 2014; Huang et al., 2015; Grady et al., 2016). According to the "early-in-late-out" hypothesis, increases in fiber tract white matter density and axonal myelination are important for cognitive development during childhood and adolescence (Paus et al., 1999). In contrast, demyelination and a loss of nerve fibers contribute to cognitive decline (Peters, 2002). Despite these early hypotheses and studies, available data describing age-related changes in structural connectivity remains lacking (Damoiseaux, 2017).

While limited, currently available evidence suggests that old age is associated with lower connectivity and lower local efficiency (Damoiseaux et al., 2007; Gong et al., 2009; Burzynska et al., 2010; Otte et al., 2015). These studies, however, address coarsely divided age subgroups. In addition, recent evidence from Zhao et al. (2015) also suggests that the age-related trajectories of local and global structural network efficiency changes are nonlinear. The use of large age ranges may then combine subjects at different stages, complicating the study of age-related structural connectivity changes that would otherwise provide useful insights for patient diagnosis and management.

Several methods have been developed to reconstruct structural connectivity networks. Previous studies have relied heavily on diffusion tensor imaging (DTI) methods to trace white matter connections (Gong et al., 2009; Betzel et al., 2014; Otte et al., 2015; Zhao et al., 2015). Conventional DTI, however, struggles to resolve complex fiber populations when they occur within a DWI voxel, as is the case when tracts cross, branch, merge, or kiss (Tuch, 2004; Hess et al., 2006; Poupon et al., 2008). As approximately one- to two-thirds of DWI voxels contain multiple fiber populations (Duarte-Carvajalino et al., 2014), structural connectivity networks reconstructed based on diffusion tensor may deviate largely from real situations. It is then necessary to develop methods capable of parsing these complex white matter structures.

In this study, age-related changes in structural connectivity were investigated using a novel Ensemble Average Propagator (EAP)-based probabilistic tractography method. Unlike DTI or orientation distribution function methods, EAP preserves the radial part of the diffusion signal and thus may accurately identify the crossing orientations of neural fascicles within a white matter voxel (Fick et al., 2014; Paquette et al., 2015). The aim of this study was to evaluate the novel EAP approach for reconstructing structural connectivity networks, using a healthy subject dataset that covered a wide range of ages.

\section{MATERIALS AND METHODS}

\section{DWI Dataset}

The lifespan datasets publicly available from the Human Connectome Project (HCP) (Marcus et al., 2011; Van Essen et al., 2013) and the OASIS3 database (Longitudinal neuroimaging, clinical, and cognitive dataset for normal aging and Alzheimer's disease $^{1}$ ) were used in this study. Datasets included 48 subjects aged 8-75 years, organized into 4 age groups (Table 1). HCP subjects (34) underwent an abbreviated scan protocols similar to that used for the WU-Minn young adult HCP study (Feinberg et al., 2010). Data for each subject was collected using a 3T General Electric MR scanner, with a whole-body radiofrequency coil for signal excitation and a quadrature 8channel brain coil for the reception. The HCP acquisition protocol consisted of: (1) high resolution 3D T1-weighted SPGR sequence, with TR/TE/flip angle of $9.1 \mathrm{~ms} / 4.1 \mathrm{~ms} / 108$, acquisition matrix size $=256 \times 256 \times 320$, and slice thickness $=1 \mathrm{~mm}$; (2) DWI using a single-shot spin-echo echo-planar sequence, including 91 non-collinear encoding directions with $b$ values of 1000, 2000, $3000 \mathrm{~s} / \mathrm{mm}^{2}$, TR/TE $=12700 / 88.3 \mathrm{~ms}$, acquisition matrix size $=144 \times 168 \times 111$, slice thickness $=2.4 \mathrm{~mm}$, and voxel size $=2.4 \mathrm{~mm} \times 2.4 \mathrm{~mm} \times 2.4 \mathrm{~mm}$, along with six additional images with no diffusion sensitization $b=0$ $\mathrm{s} / \mathrm{mm}^{2}$ (b0, non-diffusion-weighted images). The remaining 14 subjects from the OASIS3 dataset were scanned using a Siemens TIM Trio 3T MRI scanner with 16-channel head coils and the scan protocols that included: (1) high-resolution 3D T1weighted GR IR with TR/TE/flip angle = $2.4 \mathrm{~s} / 3.16 \mathrm{~ms} / 8$, acquisition matrix size $=256 \times 256 \times 176$; (2) DWI using a single-shot spin-echo echo-planar sequence with TR/TE/flip angle $=9.9 \mathrm{~s} / 102 \mathrm{~ms} / 90, b$-values $=600,800,1000 \mathrm{~s} / \mathrm{mm}^{2}$, acquisition matrix size $=96 \times 96 \times 60$, slice thickness $=2 \mathrm{~mm}$, and voxel size $=1.98 \mathrm{~mm} \times 1.98 \mathrm{~mm} \times 2 \mathrm{~mm}$, wherein diffusionweighted gradients were applied along 25 directions with one

\footnotetext{
${ }^{1}$ www.oasis-brains.org
}

TABLE 1 | Lifespan datasets from HCP and OASIS3 database, including four age groups.

\begin{tabular}{lcc}
\hline Age (years) & N & Gender \\
\hline $8-15$ & 12 & 8 Female, 4 Male \\
$25-35$ & 12 & 5 Female, 7 Male \\
$45-55$ & 12 & 5 Female, 7 Male \\
$65-75$ & 12 & 6 Female, 6 Male
\end{tabular}


b0 image. The overall process for scanning and data analysis is summarized in Figure 1.

\section{Reconstruction of the Structural Connectivity Network}

Brain parcellation and reconstruction were performed using FreeSurfer (stable version 6.0.0) ${ }^{2}$ (Fischl, 2012). Parcellations for each subject were generated in the native space, based on the collected high-resolution T1-weighted MRI images. The cerebral cortex was parcellated into 68 functional regions, 34 for each hemisphere (Desikan et al., 2006). These regions were considered as the nodes in a structural connectivity graph. Lastly, the corresponding atlas was affine registered to the DWI native space for each subject. The 68 cortical regions used to construct brain SC networks are list in Appendix Table A1.

The DWI dataset was first denoised following the procedure outlined by $\mathrm{Wu}$ et al. (2019b). The topup and eddy_openmp FSL 6.0 commands (Ubuntu Linux 16.04) were then used to correct eddy distortion and motion artifact (Andersson et al., 2003; Andersson and Sotiropoulos, 2015, 2016). Briefly, these corrections were performed by first performing an affine alignment of each DWI image to the b0 image. Next, EAPs were estimated from multi-shell DWI samples using spherical polar Fourier imaging (SPFI) (Cheng et al., 2010). According to a spherical harmonics expansion, EAPs were calculated along 726 directions evenly distributed about a spherical shell (Hess et al., 2006). Diffusion orientations contained within a white matter voxel were then extracted by detecting the local EAP peaks (Wu et al., 2018), which coincide with neural fiber tracts. Probabilistic fiber tracking was then performed to obtain the connection weights between different cortical regions (Iturria-Medina et al., 2007). Under angular constraints,

${ }^{2}$ http://www.freesurfer.net/fswiki/DownloadAndInstall

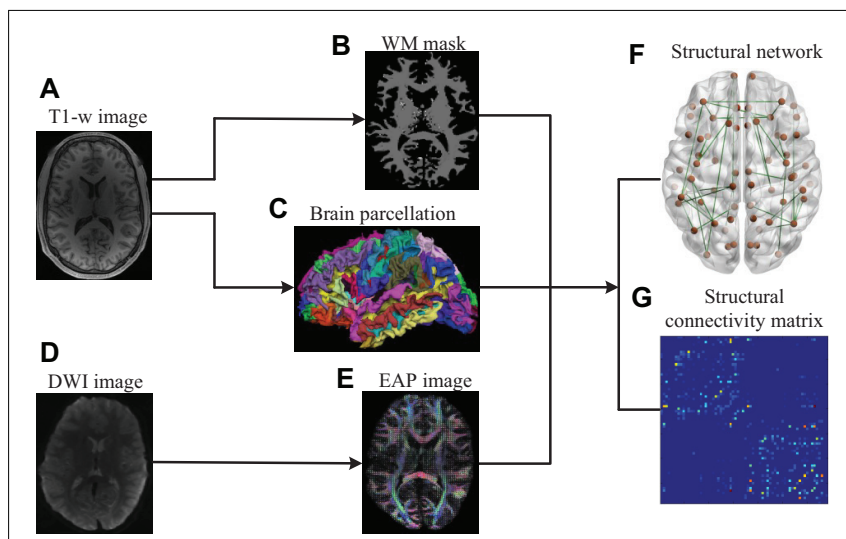

FIGURE 1 | Flowchart for the construction of structural connectivity networks based on EAP. (A) High-resolution T1-weighted MRI images. (B) White matter mask. (C) Cerebral cortex parcellation from T1-weighted MRI images using FreeSurfer. (D) DWI images. (E) EAPs constructed with SPFI. (F) Structural connectivity Networks. (G) Structural connectivity matrix. Before SC network construction, $\mathbf{( B , C )}$ are co-registered into DWI BO native space in FreeSurfer. the deterministic path planning algorithm was subsequently used to find all reasonable pathways between the WM voxels belonging to different ROIs. A train of consecutive WM voxels along each of the identified pathways was thereby determined, and the connection strengths of these pathways were computed by integrating their EAP alignment over a solid angle. The connection strength of each pair of WM voxels was then assigned as the connection strength with the largest connection possibility. Finally, the connection strength between each ROI pair was calculated as the sum of the connection probabilities for each pair of WM voxels within the ROIs (Wu et al., 2019a). The resulting link strength depended on the alignment of the EAP along connecting pathways, with higher strengths indicating better alignment. Evidence suggests that the link strengths determined by EAP fields may provide a more robust and suitable measure for structural connectivity network analysis than diffusion tensor and orientation distribution function (Assemlal et al., 2007; Cheng et al., 2010). To ensure that path propagations were anatomically realistic, we imposed a $90^{\circ}$ maximum curvature threshold between every two successive path steps (IturriaMedina et al., 2007, 2008; Sotiropoulos et al., 2010). This fiber tracking procedure yielded adjacency matrices whose elements represented the connection probabilities between each pair of parcellated cortical regions of interest.

Network graphs were created that consisted of a series of nodes connected by edges to interpret the generated adjacency matrices. Each node within a network graph represented a cortical region of interest, and the edges connecting them were assigned weights according to their determined link strength. In this study, each brain region was selected as the seed region, and its connectivity strength to each of the other 67 regions was calculated. Thus, for each subject, a $68 \times 68$ weighted, undirected network graph was constructed.

TABLE 2 | Definitions of structural connectivity network metrics.

\begin{tabular}{|c|c|}
\hline Network metrics & Description \\
\hline$E_{g l o b}(G)=\frac{1}{N(N-1)} \sum_{i \neq j \in G} \frac{1}{L_{i j}}$ & $\begin{array}{l}L_{i j} \text { is the shortest path length between node i } \\
\text { and } \mathrm{j} \text { in } \mathrm{G} \text {. }\end{array}$ \\
\hline$E_{l o c}(G)=\frac{1}{N} \sum_{i \in G} E_{g l o b}\left(G_{i}\right)$ & $\begin{array}{l}\text { Gidenotes the subgraph composed of the } \\
\text { nearest neighbors of node } \mathrm{i} \text {. }\end{array}$ \\
\hline$L_{p}(G)=\frac{1}{N(N-1)} \sum_{i \neq j \in G} L_{i j}$ & $\begin{array}{l}L_{i j} \text { is the shortest path length between node i } \\
\text { and } \mathrm{j} \text {. This function quantifies the ability for } \\
\text { information to be propagated in parallel. }\end{array}$ \\
\hline$C_{p}=\frac{1}{N} \sum_{i \in G} C(i)$ & $\begin{array}{l}C_{p} \text { is the average of the clustering coefficient } \\
\text { over all nodes, which indicates the extent of } \\
\text { local interconnectivity or cliquishness in a } \\
\text { network. }\end{array}$ \\
\hline$\gamma=C_{p}^{S C} / C_{p}^{\text {rand }}$ & $\begin{array}{l}C_{p}^{r a n d} \text { is the mean } C_{p} \text { of } 100 \text { matched random } \\
\text { networks. } C_{p}^{S C} \text { is the clustering coefficient } \\
\text { empirical SC network. }\end{array}$ \\
\hline$\lambda=L_{p}^{S C} / L_{p}^{\text {rand }}$ & $\begin{array}{l}L_{p}^{\text {rand }} \text { is the mean } L_{p} \text { of } 100 \text { matched random } \\
\text { networks. } L_{p}^{S C} \text { is the shortest length of the SC } \\
\text { networks. }\end{array}$ \\
\hline$E_{\text {nodal }}(i)=\frac{1}{N-1} \sum_{i \neq j \in G} \frac{1}{L_{i j}}$ & $\begin{array}{l}E_{\text {nodal }}(i) \text { measures the average shortest } \\
\text { pathway length between a given node } i \text { and all } \\
\text { of the other nodes in the network. }\end{array}$ \\
\hline
\end{tabular}



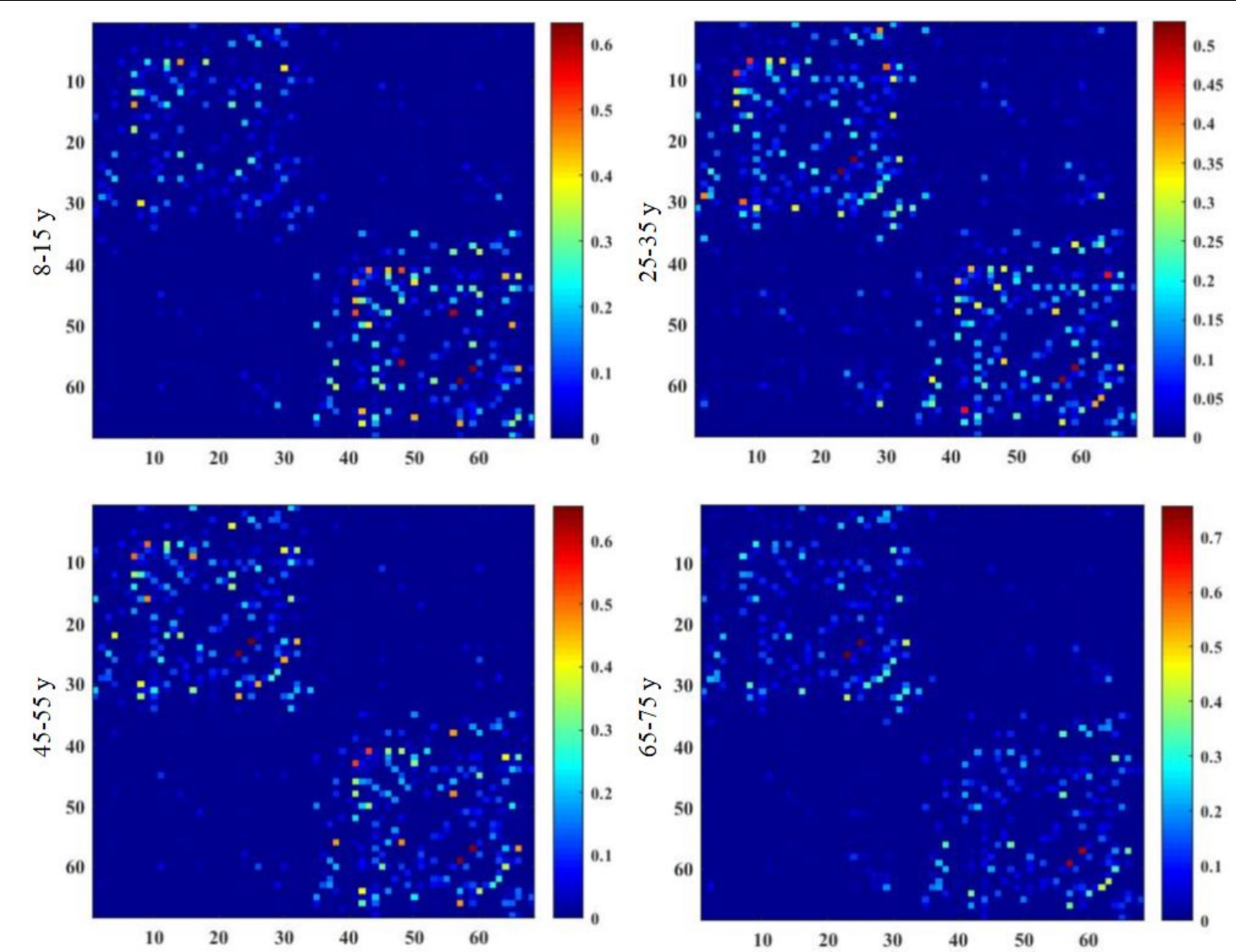

FIGURE 2 | Group-averaged structural connectivity maps of HCP lifespan subjects, which are $68 \times 68$ symmetric matrices. The subjects are arranged into four groups by age, including 8-15, 25-35, 45-55, and 65-75 years groups. In the maps, self-connections are excluded. The numbers on the axes of connectivity matrix are the indexes of parcellated regions.

\section{Structural Connectivity Network Topological Analysis}

A number of network metrics were adopted to quantify the topological features of the generated network graphs, including global efficiency $E_{\text {glob }}$, local efficiency $E_{l o c}$, clustering coefficient $C_{p}$, regional efficiency $E_{\text {nodal }}$, and the small-world parameters $\gamma$ and $\lambda$ (Cao et al., 2013). After adjacency matrices were acquired and thresholded to remove weak connections, the enumerated network metrics were computed for each age group using the GRETNA (Wang et al., 2015) and BCT toolboxes (Rubinov and Sporns, 2010). Of particular interest here are the "smallworld" parameters, which estimate the efficiency of information transfer within a defined network structure. An ideal, smallworld network described by these parameters should feature a minimum path length between any pair of nodes equal to that of a comparable random network, with greater local interconnectivity or cliquishness ( $\mathrm{He}$ et al., 2007). Lastly, the nodal efficiency metric, $E_{\text {nodal }}$, was used to describe regional properties. The mathematical equations for these measures are provided in Table 2, and detailed descriptions of each are provided by Cao et al. (2013). The weights were thresholded from 0.05 and 0.21 in intervals of 0.02 to remove spurious connections. The information from these serial measurements was integrated to calculate the area under the curve (AUC) for each metric, such that the resultant AUC values summarized the topological organization of the brain structural connectivity networks, independent of a single threshold selection. To identify the differences across age groups, we performed Kruskal-Wallis tests on the lifespan subjects. Finally, quadratic regression using least square fitting was performed to fit the AUC values of each metric.

\section{Age-Related Changes}

To further investigate age-associated structural connectivity differences, the group-wise structural connectivity hub regions were identified for each group. These are defined as regions that critically enable efficient neuronal signaling and communication 

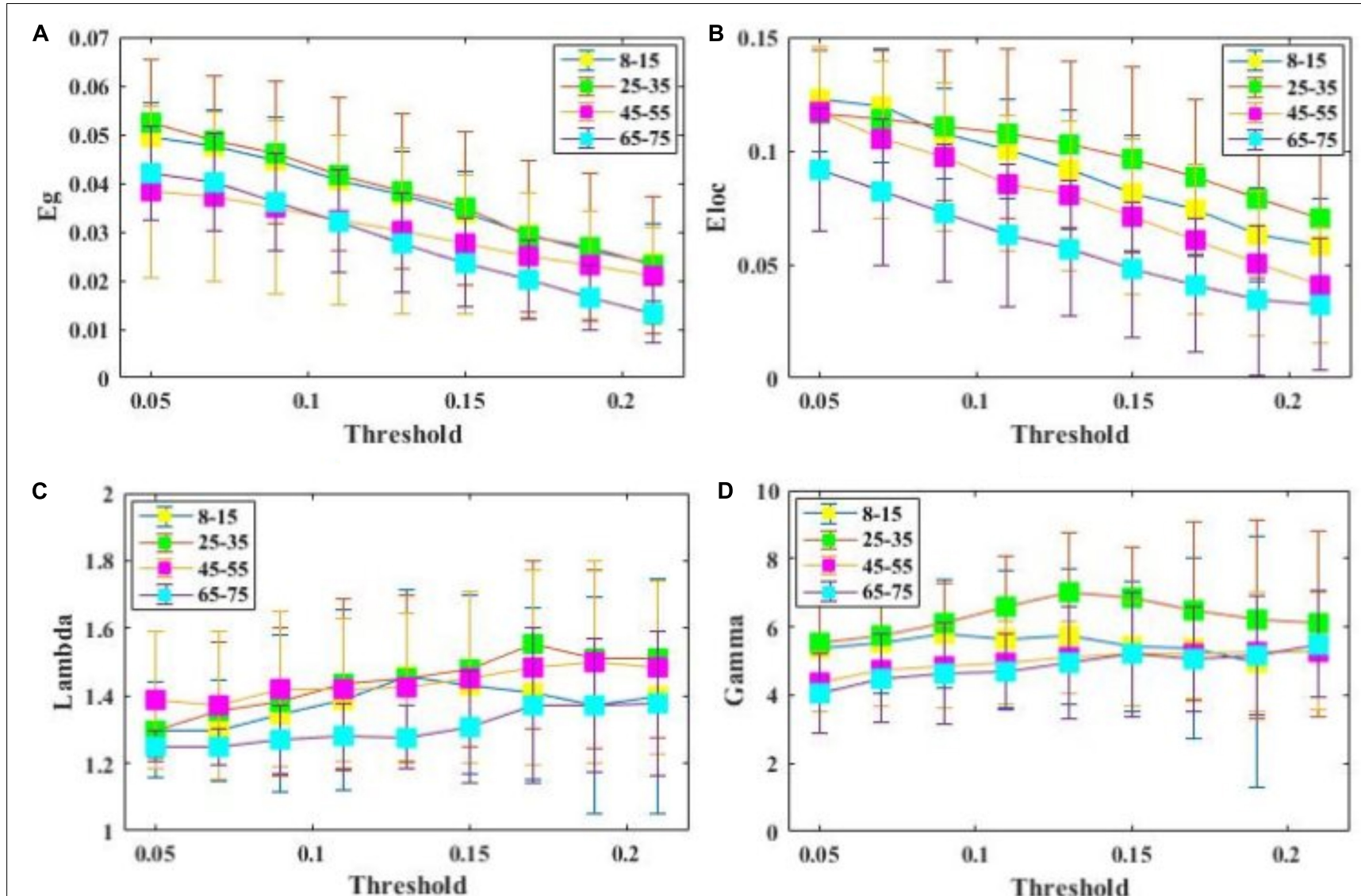

E
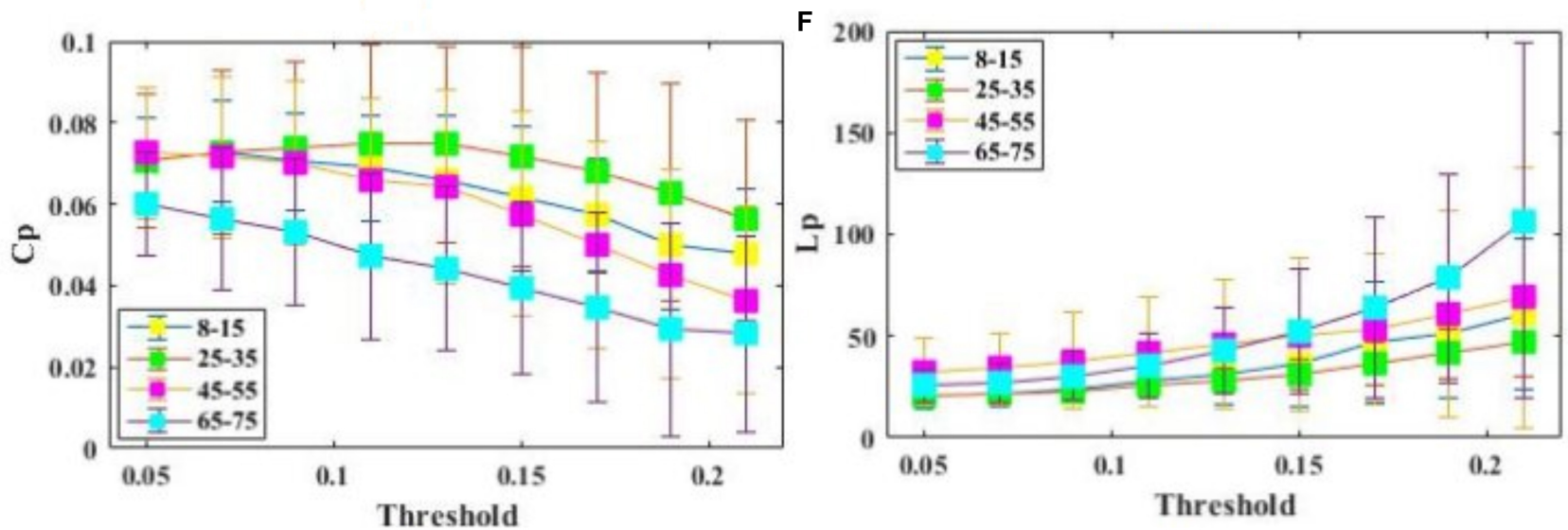

FIGURE 3 | Comparison of global and regional network metrics across 4 age groups. (A) Global efficiency $E_{\text {glob }}$, and it measures the global efficiency of parallel information transfer in a network. (B) Local efficiency $E_{l o c}$, and it measures how efficient communication is among the first neighbors of a given node when it is removed. (C) $\lambda=L_{p}^{S C} / L_{p}^{S C} L_{p}^{\text {rand }}$. (D) $\gamma=L_{p}^{S C} / L_{p}^{\text {rand }}$. (E) Clustering coefficient $C_{p}$. (F) Characterized shortest path length of SC network $L_{p}$.

TABLE 3 | Group-wise comparisons of AUC values of global and local network metrics.

\begin{tabular}{|c|c|c|c|c|c|c|}
\hline Age/y & $a E_{g l o b}$ & $a E_{l o c}$ & $a L_{p}$ & $a C_{p}$ & $a \lambda$ & $a \gamma$ \\
\hline 25-35 & $0.0061 \pm 0.0024$ & $0.0126 \pm 0.0049$ & $4.8403 \pm 1.1238$ & $0.0112 \pm 0.0037$ & $0.2301 \pm 0.0344$ & $1.0163 \pm 0.2300$ \\
\hline $65-75$ & $0.0045 \pm 0.0014$ & $0.0092 \pm 0.0045$ & $7.9390 \pm 1.4663$ & $0.0070 \pm 0.0031$ & $0.2209 \pm 0.0305$ & $0.9345 \pm 0.2775$ \\
\hline
\end{tabular}

These values were the fitted AUC values (mean $\pm S D$ ). 

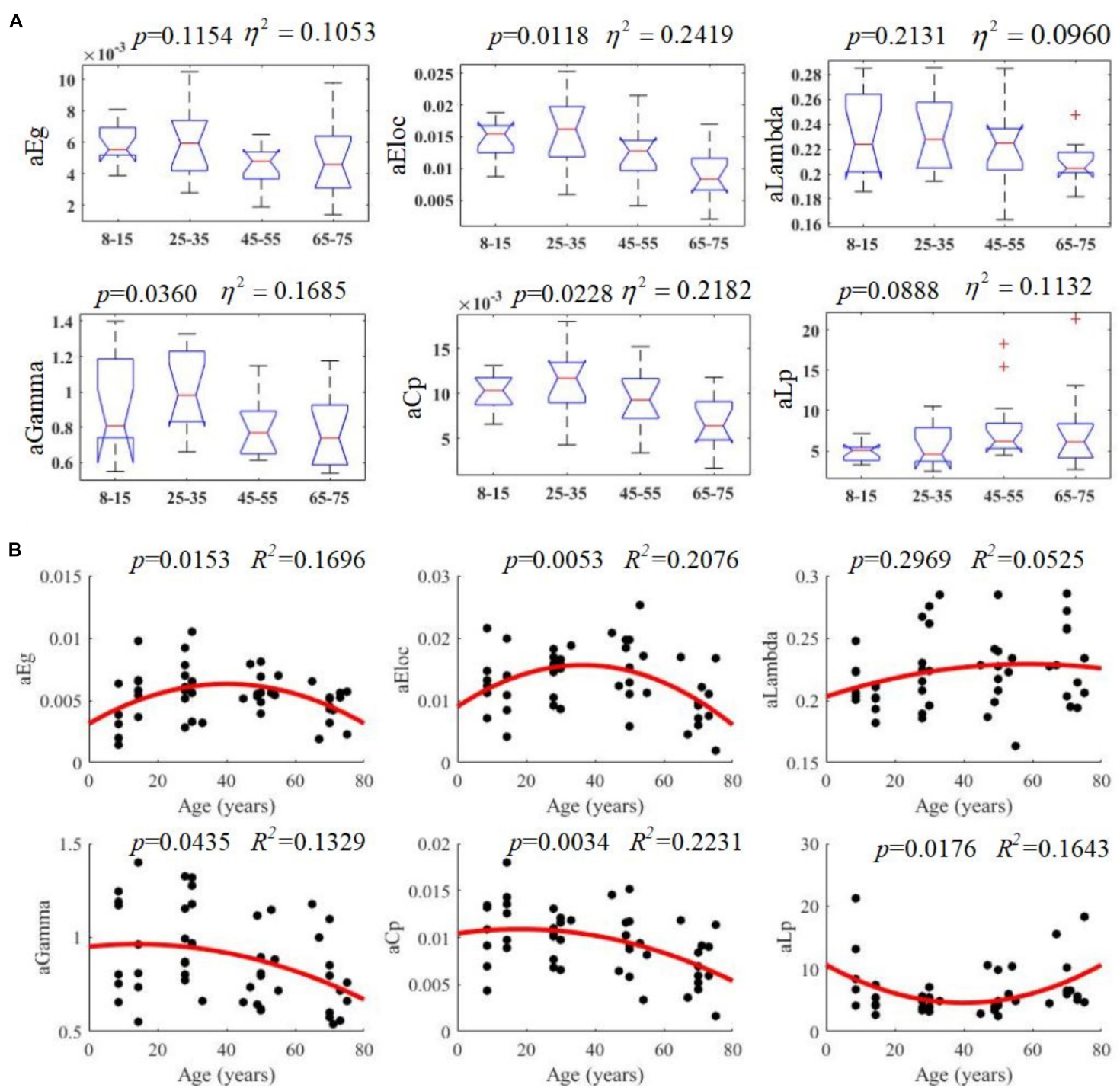

FIGURE 4 | Age-related changes in structural connectivity networks. (A) Kruskal-Wallis tests were performed to examine topological differences across age groups, and aEloc, aGamma and aCp can significantly differentiate age groups ( $p$-value $<0.05)$. (B) The trajectories of aEg, aEloc, and aCp are statistically significant at $p$-value $<0.05$.

(van den Heuvel and Sporns, 2013), integrating information to support complex cognitive functions. Network nodes were considered to be brain hubs if their nodal efficiency $E_{\text {nodal }}$ was at least one standard deviation greater than the average nodal efficiency of the network. Group-wise backbone links were also extracted from structural connectivity networks, which were formed based on the rich club connections for high-capacity brain communication (van den Heuvel et al., 2012).

\section{RESULTS}

Brain structural connectivity networks were constructed for 48 subjects using probabilistic EAP-based tractography, including 68 nodes (Appendix Table A1). Figure 2 shows the consensus structural connectivity maps for each age group. These adjacency matrices were symmetric, with self-connections excluded. The values in these maps were normalized to $[0,1]$, with higher values indicating stronger anatomical connectivity. Based on these adjacency matrices, group-wise topological properties were examined to find the structural connectivity differences between age groups.

\section{Network Metrics}

All examined groups showed a small-world organization, characterized by $\gamma>>1$ and $\lambda>1$ (Figures 3C,D). Compared with other groups, the subjects aged 65-75 years had significantly decreased $E_{\text {glob }}, E_{l o c}$, and $C_{p}$, along with increased $L_{p}$ (Figures 3A,B,E,F). The descriptive statistics for the AUC values of the thresholded graph metrics are provided in Table 3. 


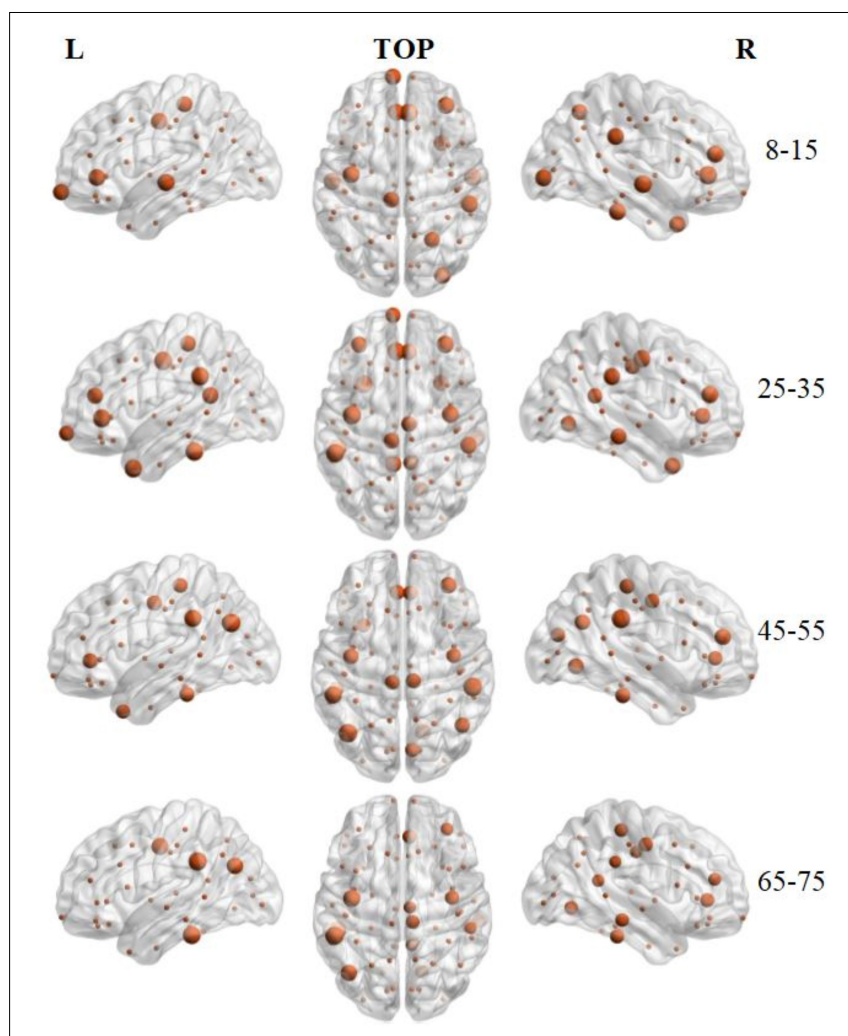

FIGURE 5 | 3D distribution of hub regions in the structural connectivity networks of the four age groups. The hub nodes are shown in brown with node sizes indicating their nodal efficiency values. The hub regions were mapped onto the cortical surface at the left, top, and right views. For the index of hub nodes, see Table 4. The brain graphs were visualized by using BrainNet Viewer software (Xia et al., 2013).

Kruskal-Wallis tests indicate that $E_{l o c}, C_{p}$, and $\gamma$ significantly differentiate the four age groups ( $p$-value $<0.05$ ) (Figure 4A). All metrics show a parabolic relationship with age from childhood to old age (Figure 4B), although the $R^{2}$ values (5.25-22.31\%) were relatively low.

\section{Hub Regions}

Hub regions were identified from the SC networks of each group. The nodes were considered as network hubs if their nodal efficiencies were at least one standard deviation greater than the average nodal efficiency of the network (Cao et al., 2013). The four age groups showed highly similar hub distributions, with core regions mainly in the frontal (region indices: 25, 59, 63) and parietal cortices (region indices: 23, 57, 64, 66) (Desikan et al., 2006), consistent with previous structural connectivity network studies on healthy adults (Sporns, 2013; van den Heuvel and Sporns, 2013). Group-wise analysis using the GRETNA toolbox then revealed that the 65-75 age group had reduced nodal efficiency in the inferior parietal (left hemisphere), inferior temporal (left hemisphere), middle temporal (left hemisphere), precuneus (left and right hemispheres), inferior parietal (right hemisphere), lateral occipital (right hemisphere), and superior parietal (right hemisphere) cortices (Figure 5 and Table 4).

\section{Backbone Links}

The number of brain structural backbone links in each age group is shown across threshold values in Figure 6, with the specific brain backbone links for each age group at a threshold of 021 shown in Figure 7. Results suggest that the number of backbone links in SC networks of the 65-57 years group significantly decreased as thresholds increased (Figure 6). However, the average backbone connection strengths showed no significant differences between age groups (Table 5).

\section{DISCUSSION}

The performance of humans on formal cognitive testing and real world cognitive function declines with aging, generally peaking in the 20 s and lessening thereafter. Understanding these changes will be critical for gaining insights into the ageassociated risk of multiple neurodegenerative disorders and the increased sensitivity of people with aging to various insults, such as infections and brain trauma. Understanding these underlying mechanisms is a first step toward treating or preventing them. Studying structural connectivity may provide vital information for understanding these normal processes of the aging brain and hence its increased vulnerability over the lifespan.

Heretofore, many studies have relied on traditional reconstruction methods that show limited performance when applied to complex fiber populations. In this preliminary study, the performance of a novel Ensemble Average Propagatorbased probabilistic tractography method was evaluated as it reconstructed the brain structural connectivity networks of 48 subjects obtained from the HCP and OASIS3 databases. The results suggest a non-linear evolution of age-dependent brain structural connectivity network properties.

The current investigation presents a novel application of the EAP. Previous EAP methods have been used to improve the diagnosis of Parkinson's disease, stroke detection, and brain tissue assessment (Banerjee et al., 2016; Brusini et al., 2016; Zucchelli et al., 2016); however, this is its first use at addressing structural connectivity. The current probabilistic method was modified from Iturria-Medina et al. (2007), extracting diffusion directions from EAP and avoiding exhaustive searches to identify the strongest paths between different ROIs.

Results obtained through this approach suggest that critical brain structural connectivity properties are conserved throughout the development process, which supports the conclusion that small-world networks are resilient to developmental alteration (Hagmann et al., 2008; Gong et al., 2009; He et al., 2009; Supekar et al., 2010). Despite retaining a small-world topology, however, the 65-75 years subjects showed decreased global efficiency, local efficiency, and increased path length (Figure 3). These findings may reflect structural degeneration due to neuron death or fiber breakdown. These results are supported by a recent study that reported reduced global efficiency in the functional brain networks of older adults (Damoiseaux, 2017). Another study, however, found no differences in global efficiency (Gong et al., 2009). Lack of differences may be ascribed to the use of a diffusion tensor 
TABLE 4 | Hub regions distributed in SC networks of the four age groups.

\begin{tabular}{|c|c|c|c|c|c|c|c|}
\hline \multicolumn{2}{|l|}{$8-15$} & \multicolumn{2}{|l|}{$25-35$} & \multicolumn{2}{|l|}{ 45-55 } & \multicolumn{2}{|l|}{$65-75$} \\
\hline Hub regions (index) & $a E_{\text {nodal }}$ & Hub regions (index) & $\mathrm{a} E_{\text {nodal }}$ & Hub regions (index) & $a E_{\text {nodal }}$ & Hub regions (index) & $a E_{\text {nodal }}$ \\
\hline 16 & 0.0082 & 8 & 0.0103 & 7 & 0.0118 & 7 & 0.0130 \\
\hline 23 & 0.0087 & 9 & 0.0092 & 8 & 0.0089 & 8 & 0.0143 \\
\hline 25 & 0.0088 & 16 & 0.0088 & 16 & 0.0087 & 23 & 0.0136 \\
\hline 29 & 0.0091 & 23 & 0.0105 & 23 & 0.0092 & 30 & 0.0148 \\
\hline 31 & 0.0084 & 25 & 0.0102 & 25 & 0.0089 & 42 & 0.0105 \\
\hline 42 & 0.0087 & 26 & 0.0089 & 30 & 0.0107 & 43 & 0.0087 \\
\hline 44 & 0.0086 & 30 & 0.0104 & 32 & 0.0082 & 46 & 0.0100 \\
\hline 59 & 0.0097 & 31 & 0.0083 & 38 & 0.0086 & 48 & 0.0100 \\
\hline 60 & 0.0089 & 32 & 0.0101 & 41 & 0.0092 & 50 & 0.0100 \\
\hline 62 & 0.0081 & 43 & 0.0082 & 42 & 0.0090 & 56 & 0.0087 \\
\hline 63 & 0.0093 & 46 & 0.0086 & 46 & 0.0096 & 57 & 0.0107 \\
\hline 64 & 0.0087 & 48 & 0.0090 & 50 & 0.0089 & 59 & 0.0104 \\
\hline \multirow[t]{6}{*}{66} & 0.0082 & 56 & 0.0085 & 57 & 0.0086 & 60 & 0.0091 \\
\hline & & 57 & 0.0097 & 59 & 0.0084 & 64 & 0.0083 \\
\hline & & 59 & 0.0089 & 60 & 0.0099 & & \\
\hline & & 60 & 0.0097 & 64 & 0.0119 & & \\
\hline & & 64 & 0.0094 & & & & \\
\hline & & 66 & 0.0089 & & & & \\
\hline
\end{tabular}

The nodes were considered brain hubs if their nodal efficiencies were at least 1 SD greater than the average nodal efficiency of the network. The corresponding indexes (see Appendix Table A1) are listed.

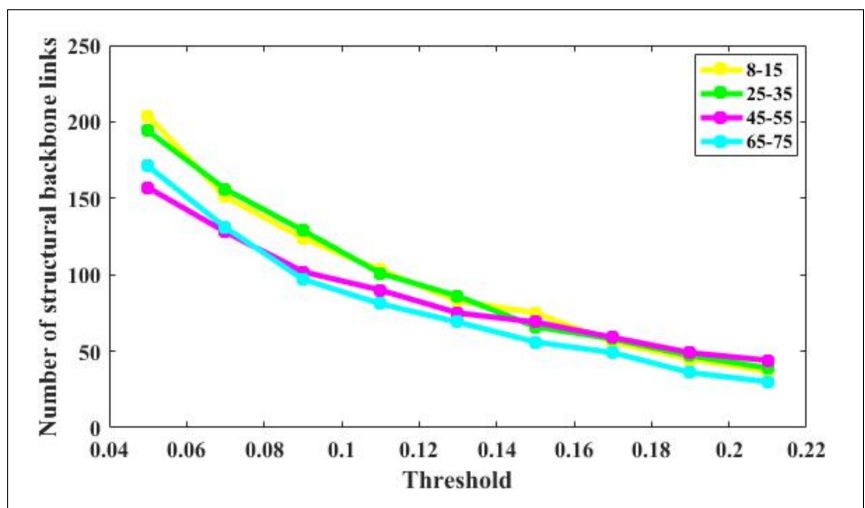

FIGURE 6 | Number of backbone links of the structural connectivity networks of the four age groups. For the $65-75$ group, the number of backbone links is decreases significantly. For other groups, there are some minor differences.

model neural tracking that cannot resolve multiple fiber populations in a DWI voxel.

To examine the topological changes that occur over the lifespan here, a quadratic regression model was fit to the AUC values of each network metric (Figure 4). These results suggest that the lifetime development of structural cortical networks follows a non-linear trajectory. From 7 to 35 years, brain networks strengthen with growth, training, and learning. From 35 to 75 years, however, the network is gradually reduced as overall cortical connectivity declines. These results are mostly consistent with a recent study performed by Zhao et al. (2015), which found a similar non-linear trajectory of structural changes. The present study then investigated the patterns of specific network topological properties. In this way, global efficiency, local efficiency, small-worldness, and cluster coefficients were found to follow negative parabolic trajectories, while characteristic pathway lengths followed a positive parabolic trajectory.

These findings complement the negative parabolic trajectories that were uncovered for the global network properties (Zhao et al., 2015), including network strength, cost, topological efficiency, and robustness. Together, these results suggest that the overall shape of graph network trajectories is consistent and that SC network changes during development and aging are nonlinear. These results, then, identify possible structural substrates underlying functional and cognitive changes during development and aging and may be important for separating pathogenic changes from normal aging processes. Some minor differences between these two studies may arise from differences in the dataset, tractography method, and brain parcellation schemes. The current EAP-based probabilistic tractography method has shown to improve fiber tracking accuracy (Descoteaux et al., 2009). Given the scarcity of data, future studies will be required to further corroborate these important findings.

To identify the hub regions, we examined the nodal efficiency of each cortical region. In the 8-15 age group, 13 regions were identified as the hubs by their large nodal efficiency $E_{\text {nodal }}$ values. In the 25-35 and 45-55 age groups, 18 and 16 regions were identified as the hubs, respectively, and 14 regions were identified as hubs in the 65-75 age group (Table 4). Identified hubs were predominately located in regions of the frontal and parietal cortices that connected with multiple other cortical regions (Mesulam, 1998). This dense interconnectivity suggests their pivotal roles in the human structural cortical networks. 


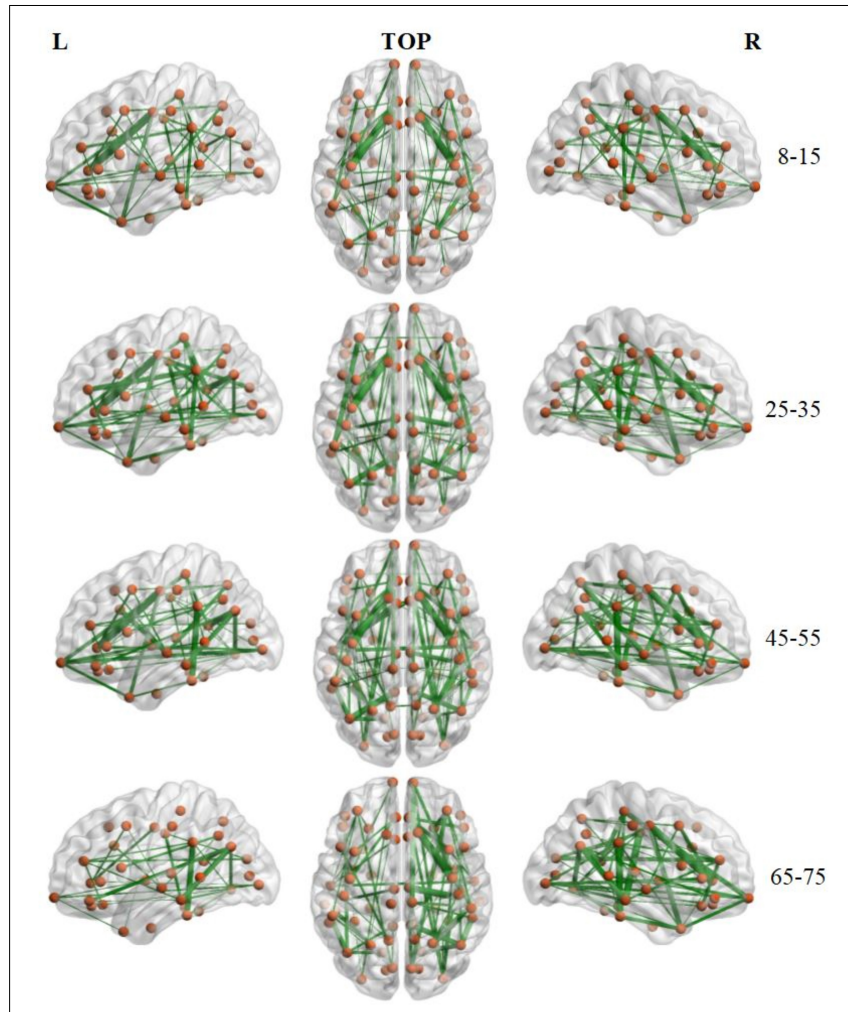

FIGURE 7 | Backbone connections of brain structural connectivity networks of four groups at the strength threshold of 0.21 . The links were mapped into the brain at the left, top, and right views. The edge widths represent the connection weights between nodes. The brain graphs were visualized by using BrainNet Viewer software (Xia et al., 2013).

The findings align with several previous studies, in which these cortical regions were identified as critical nodes in both structural and functional brain networks in humans (Achard et al., 2006; He et al., 2007). Finally, it should be noted that only the 3rd and 4th age groups included data from both the OASIS3 and HCP datasets. Consequently, these groups included subjects who were scanned using different systems with different acquisition parameters. Although the overall data showed the same trends, the effect that these protocol variations may have had on network construction remains to be studied.

Backbone connections were then investigated across age groups. Although all age groups showed decreases in the backbone number as the threshold increased, the number of backbone connections in the 65-75 years group showed a particularly noteworthy reduction (Figure 6). The connections that survived at the highest threshold value were primarily composed of the prefrontal cortex and the insular cortex (Figure 7). These results suggest a strong correlation between the SC wiring within the prefrontal and insular cortex and the behavioral symptoms of old age, such as inattention and amnesia. While the adoption of a lower threshold may provide a more comprehensive snapshot of the structural network, probability-based approaches carry the possibility of identifying spurious nerve fiber connections between regions that are not biologically connected. The use of a high probability threshold may assuage this issue and suggests that present results are not dependent on an arbitrarily chosen threshold. To ensure that spurious connections were removed, a range of thresholds were applied between 0.05 and 0.21 in intervals of 0.02 . Links whose connection strength fell below the chosen threshold were excluded from topological analysis ensuring that only strong connections were retained. Despite the tested range, no threshold value caused a significant change in the non-linear patterns of age-dependent brain structural connectivity networks.

The human brain undergoes both macro- and micro-scale structural changes throughout its lifespan (Lebel and Deoni, 2018). The white matter plasticity in response to learning or environmental stimuli can alter the brain's structural connectivity network, although the magnitude and time course of these changes can vary. Comprehensive lifespan studies provide valuable insight into the processes of macrostructural brain changes and have contributed to our understanding of brain development (Gong et al., 2009; Hagmann et al., 2010; Betzel et al., 2014; Otte et al., 2015; Zhao et al., 2015). The large-scale brain changes during development provide a context for studying white matter changes at the macroscopic level. The current work aimed to characterize normative brain development from 8 to 75 years, emphasizing group-wise comparative studies and statistical techniques to study structural connectivity network development. Previous studies have demonstrated consistent, rapid white matter development over the first 3 years of life, suggesting increased myelination and axonal packing (Huang et al., 2013). Results here clearly demonstrates that the structural connectivity network continually strengthens during later childhood, adolescence, and adulthood, due to white matter changes.

There are several limitations to this study. First, this study had a relatively small sample size $(N=48)$ and results should be interpreted in that context. Nevertheless, the parabolic patterns of functional changes observed using the novel EAP method are promising, suggesting that an EAP-based method is a reliable tool

TABLE 5 | Connection strength of backbone links at different thresholds.

\begin{tabular}{|c|c|c|c|c|c|}
\hline & $>0.05$ & $>0.09$ & $>0.13$ & $>0.17$ & $>0.21$ \\
\hline $8-15$ & $0.1414 \pm 0.0947$ & $0.1900 \pm 0.0932$ & $0.2295 \pm 0.0904$ & $0.2660 \pm 0.0892$ & $0.3066 \pm 0.0842$ \\
\hline $25-5$ & $0.1556 \pm 0.1157$ & $0.1993 \pm 0.1198$ & $0.2457 \pm 0.1225$ & $0.2947 \pm 0.1218$ & $0.3467 \pm 0.1172$ \\
\hline $45-55$ & $0.1675 \pm 0.1200$ & $0.2207 \pm 0.1183$ & $0.2603 \pm 0.1143$ & $0.2904 \pm 0.1109$ & $0.3254 \pm 0.1077$ \\
\hline $65-75$ & $0.1409 \pm 0.1050$ & $0.1956 \pm 0.1115$ & $0.2304 \pm 0.1150$ & $0.2657 \pm 0.1195$ & $0.3154 \pm 0.1303$ \\
\hline
\end{tabular}

These values are probability values (mean $\pm S D$ ) of links in each group. 
for future studies. These preliminary findings will require further corroboration from future studies with larger-sample sizes. Second, a probabilistic tractography method based on EAP was used to construct whole-brain neural connectivity, which is more capable of tracking complicated fiber tracts than deterministic tractography methods (Behrens et al., 2007). While this provides a better reconstruction of the brain structural network, the reliance on a probabilistic method allows for connections to be drawn between regions that are not biologically linked. Spurious connections are likely to show limited connection strength, however, so the adoption of a high probability threshold should minimize this issue, and the number of remaining connections further suggests that results were not dependent on an arbitrarily chosen threshold.

Third, the whole-brain structural connectivity networks were only derived from DWI data. The brain networks can also be studied using both structural and functional MRI data. The combination of multimodal neuroimaging techniques should add considerably to our understandings of how age-linked structural disruptions in neuronal circuits are associated with functional alterations. Lastly, subjects from the different datasets (HCP and OASIS3) were collected using different scanners and different acquisition protocols, the effects of which are currently unknown and require further study. Although T1weighted images were denoised through a non-local SVD method (Wu et al., 2019b), alleviating the possible influences of white matter hyperintensities (WMH) on the WM segmentation, future investigations will be necessary to understand the extent to which WMHs could affect EAP calculation (Popescu et al., 2014; Valverde et al., 2014; Prados et al., 2016). Finally, several studies have suggested that the graphic metrics of whole-brain structural connectivity networks are heavily dependent on the resolution of their cortex parcellation (Wang et al., 2009). In the future, different parcellation schemes should be used in conjunction to provide a holistic investigation of topological SC network features.

\section{CONCLUSION}

Non-linear parabolic patterns of age-dependent brain structural connectivity network properties were observed across the age ranges of 8-75 years using a probabilistic Ensemble Average Propagator-based tractography method. This novel method provided a reliable method to construct macroscale structural connectivity networks that capture age-associated changes in brain structure. This study reveals new insights into age

\section{REFERENCES}

Achard, P., Cheng, H., De Grauwe, L., Decat, J., Schoutteten, H., Moritz, T., et al. (2006). Integration of plant responses to environmentally activated phytohormonal signals. Science 311, 91-94. doi: 10.1126/science.1118642

Anderson, A. W. (2005). Measurement of fiber orientation distributions using high angular resolution diffusion imaging. Magn. Reson. Med. 54, 1194-1206. doi: $10.1002 / \mathrm{mrm} .20667$

Andersson, J. L., Skare, S., and Ashburner, J. (2003). How to correct susceptibility distortions in spin-echo echo-planar images: application to diffusion related changes in cognitive function that are observed clinically and starts the process of helping us understand where to focus our efforts at understanding the very important, agerelated brain changes that markedly increase vulnerability to neurodegenerative disorders. Understanding age-related vulnerability, in turn, will help us design methods to reduce these age related risks.

\section{DATA AVAILABILITY STATEMENT}

The datasets generated for this study are available on request to the corresponding author.

\section{ETHICS STATEMENT}

Ethical review and approval was not required for the study on human participants in accordance with the local legislation and institutional requirements. The patients/participants provided their written informed consent to participate in this study.

\section{AUTHOR CONTRIBUTIONS}

ZW conducted the data analysis, result summarization, and manuscript drafting. YP contributed to study design, result preparation, and manuscript revising. SS and PS contributed to study design and manuscript revising with clinical knowledge. YZ contributed to study design, result review, manuscript revising, and finalization.

\section{FUNDING}

The research was supported by Natural Science Foundation of Zhejiang Province (LY20E070005 and LY17E070007), China, National Natural Science Foundation of China (51207038), and the University of Houston. Thirty four datasets were provided by Human Connectome Project, WU-Minn Consortium (Principal Investigators: David Van Essen and Kamil Ugurbil; 1U54MH091657) funded by the $16 \mathrm{NIH}$ Institutes and Centers that support the NIH Blueprint for Neuroscience Research; and by the McDonnell Center for Systems Neuroscience at Washington University. Other 14 datasets were selected from OASIS3 project (P50 AG05681, P01 AG03991, R01 AG021910, P50 MH071616, U24 RR021382, and R01 MH56584).

tensor imaging. Neuroimage 20, 870-888. doi: 10.1016/s1053-8119(03) 00336-7

Andersson, J. L., and Sotiropoulos, S. N. (2015). Non-parametric representation and prediction of single-and multi-shell diffusion-weighted MRI data using Gaussian processes. Neuroimage 122, 166-176. doi: 10.1016/j.neuroimage. 2015.07.067

Andersson, J. L., and Sotiropoulos, S. N. (2016). An integrated approach to correction for off-resonance effects and subject movement in diffusion MR imaging. Neuroimage 125, 1063-1078. doi: 10.1016/j.neuroimage.2015. 10.019 
Assemlal, H. E., Tschumperlé, D., and Brun, L. (2007). "Fiber tracking on HARDI data using robust ODF fields," in Proceedings of the IEEE International Conference on Image Xplore, San Antonio, TX, 133-136.

Banerjee, M., Okun, M. S., Vaillancourt, D. E., and Vemuri, B. (2016). A method for automated classification of Parkinson's disease diagnosis using an Ensemble Average Propagator template brain map estimated from diffusion MRI. PLoS One 11:e0155764. doi: 10.1371/journal.pone.0155764

Bassett, D. S., Brown, J. A., Deshpande, V., Carlson, J. M., and Grafton, S. T. (2011). Conserved and variable architecture of human white matter connectivity. Neuroimage 54, 1262-1279. doi: 10.1016/j.neuroimage.2010.09.006

Behrens, T. E., Berg, H. J., Jbabdi, S., Rushworth, M. F., and Woolrich, M. W. (2007). Probabilistic diffusion tractography with multiple fibre orientations: what can we gain? Neuroimage 34, 144-155. doi: 10.1016/j.neuroimage.2006. 09.018

Betzel, R. F., Byrge, L., He, Y., Goñi, J., Zuo, X. N., and Sporns, O. (2014). Changes in structural and functional connectivity among resting-state networks across the human lifespan. Neuroimage 102, 345-357. doi: 10.1016/j.neuroimage.2014. 07.067

Brusini, L., Obertino, S., Galazzo, I. B., Zucchelli, M., Krueger, G., Granziera, C., et al. (2016). Ensemble average propagator-based detection of microstructural alterations after stroke. Int. J. Comput. Assist. Radiol. Surg. 11, 1585-1597. doi: $10.1007 / \mathrm{s} 11548-016-1442-\mathrm{z}$

Burzynska, A. Z., Preuschhof, C., Bäckman, L., Nyberg, L., Lim, S. C., Lindenberger, U., et al. (2010). Age-related differences in white matter microstructure: regionspecific patterns of diffusivity. Neuroimage 49, 2104-2112. doi: 10.1016/j. neuroimage.2009.09.041

Cao, Q., Shu, N., An, L., Wang, P., Sun, L., Xia, M. R., et al. (2013). Probabilistic diffusion tractography and graph theory analysis reveal abnormal white matter structural connectivity networks in drug-naive boys with attention deficit/hyperactivity disorder. J. Neurosci. 33, 10676-10687. doi: 10.1523/ jneurosci.4793-12.2013

Cheng, J., Ghosh, A., Jiang, T., and Deriche, R. (2010). "Model-free and analytical EAP reconstruction via spherical polar Fourier diffusion MRI," in Proceedings of the International Conference on Medical Image Computing and ComputerAssisted Intervention, (Berlin: Springer), 590-597. doi: 10.1007/978-3-64215705-9_72

Damoiseaux, J. S. (2017). Effects of aging on functional and structural brain connectivity. Neuroimage 160, 32-40. doi: 10.1016/j.neuroimage.2017. 01.077

Damoiseaux, J. S., Beckmann, C., Arigita, E. S., Barkhof, F., Scheltens, P., Stam, C., et al. (2007). Reduced resting-state brain activity in the "default network" in normal aging. Cereb. Cortex 18, 1856-1864. doi: 10.1093/cercor/bhm207

Dennis, E. L., and Thompson, P. M. (2014). Functional brain connectivity using fMRI in aging and Alzheimer's disease. Neuropsychol. Rev. 24, 49-62. doi: 10. 1093/cercor/bhu259

Descoteaux, M., Deriche, R., Knosche, T. R., and Anwander, A. (2009). Deterministic and probabilistic tractography based on complex fibre orientation distributions. IEEE Trans. Med. Imaging 28, 269-286. doi: 10.1109/TMI.2008.2004424

Desikan, R. S., Ségonne, F., Fischl, B., Quinn, B. T., Dickerson, B. C., Blacker, D., et al. (2006). An automated labeling system for subdividing the human cerebral cortex on MRI scans into gyral based regions of interest. Neuroimage 31, 968-980. doi: 10.1016/j.neuroimage.2006.01.021

Duarte-Carvajalino, J. M., Lenglet, C., Xu, J., Yacoub, E., Ugurbil, K., Moeller, S., et al. (2014). Estimation of the CSA-ODF using Bayesian compressed sensing of multi-shell HARDI. Magn. Reson. Med. 72, 1471-1485. doi: 10.1002/mrm. 25046

Feinberg, D. A., Moeller, S., Smith, S. M., Auerbach, E., Ramanna, S., Glasser, M. F., et al. (2010). Multiplexed echo planar imaging for sub-second whole brain FMRI and fast diffusion imaging. PLoS One 5:e15710. doi: 10.1371/journal. pone. 0015710

Fick, R. H., Wassermann, D., Sanguinetti, G., and Deriche, R. (2014). An analytical 3D Laplacian regularized SHORE basis and its impact on EAP reconstruction and microstructure recovery. Math. Vis. 39, 151-165. doi: 10.1007/978-3-31911182-7_14

Fischl, B. (2012). FreeSurfer. Neuroimage 62, 774-781. doi: 10.1016/j.neuroimage. 2012.01.021
Frank, L. R. (2002). Characterization of anisotropy in high angular resolution diffusion-weighted MRI. Magn. Reson. Med. 47, 1083-1099. doi: 10.1002/mrm. 10156

Geerligs, L., Renken, R. J., Saliasi, E., Maurits, N. M., and Lorist, M. M. (2014). A brain-wide study of age-related changes in functional connectivity. Cereb. Cortex 25, 1987-1999. doi: 10.1093/cercor/bhu012

Gong, G., Rosa-Neto, P., Carbonell, F., Chen, Z. J., He, Y., and Evans, A. C. (2009). Age-and gender-related differences in the cortical anatomical network. J. Neurosci. 29, 15684-15693. doi: 10.1523/JNEUROSCI.2308-09.2009

Grady, C., Sarraf, S., Saverino, C., and Campbell, K. (2016). Age differences in the functional interactions among the default, frontoparietal control, and dorsal attention networks. Neurobiol. Aging 41, 159-172. doi: 10.1016/j. neurobiolaging.2016.02.020

Hagmann, P., Cammoun, L., Gigandet, X., Meuli, R., Honey, C. J., Wedeen, V. J., et al. (2008). Mapping the structural core of human cerebral cortex. PLoS Biol. 6:e159. doi: 10.1371/journal.pbio.0060159

Hagmann, P., Sporns, O., Madan, N., Cammoun, L., Pienaar, R., Wedeen, V. J., et al. (2010). White matter maturation reshapes structural connectivity in the late developing human brain. Proc. Natl. Acad. Sci. U.S.A. 107, 19067-19072. doi: 10.1073/pnas.1009073107

He, Y., Chen, Z. J., and Evans, A. C. (2007). Small-world anatomical networks in the human brain revealed by cortical thickness from MRI. Cereb. Cortex 17, 2407-2419. doi: 10.1093/cercor/bhl149

He, Y., Wang, J., Wang, L., Chen, Z. J., Yan, C., Yang, H., et al. (2009). Uncovering intrinsic modular organization of spontaneous brain activity in humans. PLoS One 4:e5226. doi: 10.1371/journal.pone.0005226

Hess, C. P., Mukherjee, P., Han, E. T., Xu, D., and Vigneron, D. B. (2006). Q-ball reconstruction of multimodal fiber orientations using the spherical harmonic basis. Magn. Reson. Med. 56, 104-117. doi: 10.1002/mrm.20931

Huang, C. C., Hsieh, W. J., Lee, P. L., Peng, L. N., Liu, L. K., Lee, W. J., et al. (2015). Age-related changes in resting-state networks of a large sample size of healthy elderly. CNS Neurosci. Ther. 21, 817-825. doi: 10.1111/cns.12396

Huang, H., Shu, N., Mishra, V., Jeon, T., Chalak, L., Wang, Z. J., et al. (2013). Development of human brain structural networks through infancy and childhood. Cereb. Cortex 25, 1389-1404. doi: 10.1093/cercor/ bht 335

Iturria-Medina, Y., Canales-Rodriguez, E., Melie-Garcia, L., Valdes-Hernandez, P., Martinez-Montes, E., Alemán-Gómez, Y., et al. (2007). Characterizing brain anatomical connections using diffusion weighted MRI and graph theory. Neuroimage 36, 645-660. doi: 10.1016/j.neuroimage.2007.02.012

Iturria-Medina, Y., Sotero, R. C., Canales-Rodríguez, E. J., Alemán-Gómez, Y., and Melie-García, L. (2008). Studying the human brain anatomical network via diffusion-weighted MRI and Graph Theory. Neuroimage 40, 1064-1076. doi: 10.1016/j.neuroimage.2007.10.060

Lebel, C., and Deoni, S. (2018). The development of brain white matter microstructure. Neuroimage 182, 207-218. doi: 10.1016/j.neuroimage.2017.12. 097

Lerch, J. P., van der Kouwe, A. J., Raznahan, A., Paus, T., Johansen-Berg, H., Miller, K. L., et al. (2017). Studying neuroanatomy using MRI. Nat. Neurosci. 20, 314-326. doi: 10.1038/nn.4501

Maier-Hein, K. H., Neher, P. F., Houde, J. C., Côté, M. A., Garyfallidis, E., Zhong, J., et al. (2017). The challenge of mapping the human connectome based on diffusion tractography. Nat. Commun. 8:1349. doi: 10.1038/s41467-01701285-X

Marcus, D., Harwell, J., Olsen, T., Hodge, M., Glasser, M., Prior, F., et al. (2011). Informatics and data mining tools and strategies for the human connectome project. Front. Neuroinform. 5:4. doi: 10.3389/fninf.2011.00004

Mesulam, M. M. (1998). From sensation to cognition. Brain 121, 1013-1052. doi: 10.1093/brain/121.6.1013

Onoda, K., Ishihara, M., and Yamaguchi, S. (2012). Decreased functional connectivity by aging is associated with cognitive decline. J. Cogn. Neurosci. 24, 2186-2198. doi: 10.1162/jocn_a_00269

Otte, W. M., van Diessen, E., Paul, S., Ramaswamy, R., Rallabandi, V. S., Stam, C. J., et al. (2015). Aging alterations in whole-brain networks during adulthood mapped with the minimum spanning tree indices: the interplay of density, connectivity cost and life-time trajectory. Neuroimage 109, 171-189. doi: 10. 1016/j.neuroimage.2015.01.011 
Paquette, M., Merlet, S., Gilbert, G., Deriche, R., and Descoteaux, M. (2015). Comparison of sampling strategies and sparsifying transforms to improve compressed sensing diffusion spectrum imaging. Magn. Reson. Med. 73, 401416. doi: 10.1002/mrm.25093

Paus, T., Zijdenbos, A. P., Worsley, K., Collins, L., Blumenthal, J., Giedd, J. N., et al. (1999). Structural maturation of neural pathways in children and adolescents: in vivo study. Science 283, 1908-1911. doi: 10.1126/science.283.5409. 1908

Peters, A. (2002). The effects of normal aging on myelin and nerve fibers: a review. J. Neurocytol. 31, 581-593.

Popescu, V., Ran, N. C. G., Barkhof, F., Chard, D. T., Wheeler-Kingshott, C. A., and Vrenken, H. (2014). Accurate gm atrophy quantification in ms using lesionfilling with co-registered $2 \mathrm{~d}$ lesion masks. Neuroimage Clin. 4, 366-373. doi: 10.1016/j.nicl.2014.01.004

Poupon, C., Rieul, B., Kezele, I., Perrin, M., Poupon, F., and Mangin, J. F. (2008). New diffusion phantoms dedicated to the study and validation of highangular-resolution diffusion imaging (HARDI) models. Magn. Reson. Med. 60, 1276-1283. doi: 10.1002/mrm.21789

Prados, F., Cardoso, M. J., Kanber, B., Ciccarelli, O., and Ourselin, S. (2016). A multi-time-point modality-agnostic patch-based method for lesion filling in multiple sclerosis. Neuroimage 139, 376-384. doi: 10.1016/j.neuroimage.2016. 06.053

Rubinov, M., and Sporns, O. (2010). Complex network measures of brain connectivity: uses and interpretations. Neuroimage 52, 1059-1069. doi: 10. 1016/j.neuroimage.2009.10.003

Schmahmann, J. D., Pandya, D. N., Wang, R., Dai, G., D'arceuil, H. E., de Crespigny, A. J., et al. (2007). Association fibre pathways of the brain: parallel observations from diffusion spectrum imaging and autoradiography. Brain 130, 630-653. doi: 10.1093/brain/awl359

Sotiropoulos, S. N., Bai, L., Morgan, P. S., Constantinescu, C. S., and Tench, C. R. (2010). Brain tractography using Q-ball imaging and graph theory: improved connectivities through fibre crossings via a model-based approach. Neuroimage 49, 2444-2456. doi: 10.1016/j.neuroimage.2009.10.001

Sporns, O. (2013). Network attributes for segregation and integration in the human brain. Curr. Opin. Neurobiol. 23, 162-171. doi: 10.1016/j.conb.2012.11.015

Supekar, K., Uddin, L. Q., Prater, K., Amin, H., Greicius, M. D., and Menon, V. (2010). Development of functional and structural connectivity within the default mode network in young children. Neuroimage 52, 290-301. doi: 10. 1016/j.neuroimage.2010.04.009

Tuch, D. S. (2004). Q-ball imaging. Magn. Reson. Med. 52, 1358-1372.

Tuch, D. S., Reese, T. G., Wiegell, M. R., Makris, N., Belliveau, J. W., and Wedeen, V. J. (2002). High angular resolution diffusion imaging reveals intravoxel white matter fiber heterogeneity. Magn. Reson. Med. 48, 577-582. doi: 10.1002/mrm. 10268

Valverde, S., Oliver, A., and Lladó, X. (2014). A white matter lesion-filling approach to improve brain tissue volume measurements. Neuroimage Clin. 6, 86-92. doi: $10.1016 /$ j.nicl.2014.08.016 van den Heuvel, M. P., Kahn, R. S., Goñi, J., and Sporns, O. (2012). High-cost, highcapacity backbone for global brain communication. Proc. Natl. Acad. Sci. U.S.A. 109, 11372-11377. doi: 10.1073/pnas.1203593109

van den Heuvel, M. P., and Sporns, O. (2013). Network hubs in the human brain. Trends Cogn. Sci. 17, 683-696. doi: 10.1016/j.tics.2013.09.012

Van Essen, D. C., Smith, S. M., Barch, D. M., Behrens, T. E., Yacoub, E., Ugurbil, K., et al. (2013). The WU-Minn human connectome project: an overview. Neuroimage 80, 62-79. doi: 10.1016/j.neuroimage.2013.05.041

Wang, J., Wang, L., Zang, Y., Yang, H., Tang, H., Gong, Q., et al. (2009). Parcellation-dependent small-world brain functional networks: a resting-state fMRI study. Hum. Brain Mapp. 30, 1511-1523. doi: 10.1002/hbm.20623

Wang, J., Wang, X., Xia, M., Liao, X., Evans, A., and He, Y. (2015). GRETNA: a graph theoretical network analysis toolbox for imaging connectomics. Front. Hum. Neurosci. 9:386. doi: 10.3389/fnhum.2015.00386

Wu, Z., Peng, Y., Xu, D., Hong, M., and Zhang, Y. (2019a). Construction of brain structural connectivity network using a novel integrated algorithm based on ensemble average propagator. Comput. Biol. Med. 112:103384. doi: 10.1016/j. compbiomed.2019.103384

Wu, Z., Xu, D., Potter, T., and Zhang, Y. (2019b). Denoising high angular resolution diffusion imaging data by combining singular value decomposition and non-local means filter. J. Neurosci. Methods 312, 105-113. doi: 10.1016/j. jneumeth.2018.11.020

Wu, Z., Yu, X., Liu, Y., and Hong, M. (2018). A PSO-Powell hybrid method to extract fiber orientations from ODF. Comput. Math. Methods Med. 2018:7680164.

Xia, M., Wang, J., and He, Y. (2013). BrainNet Viewer: a network visualization tool for human brain connectomics. PLoS One. 8:e68910. doi: 10.1371/journal.pone. 0068910

Zhao, T., Cao, M., Niu, H., Zuo, X. N., Evans, A., He, Y., et al. (2015). Agerelated changes in the topological organization of the white matter structural connectome across the human lifespan. Hum. Brain Mapp. 36, 3777-3792. doi: 10.1002/hbm. 22877

Zucchelli, M., Brusini, L., Méndez, C. A., Daducci, A., Granziera, C., and Menegaz, G. (2016). What lies beneath? Diffusion EAP-based study of brain tissue microstructure. Med. Image Anal. 32, 145-156. doi: 10.1016/j.media.2016.03. 008

Conflict of Interest: The authors declare that the research was conducted in the absence of any commercial or financial relationships that could be construed as a potential conflict of interest.

Copyright (c) 2020 Wu, Peng, Selvaraj, Schulz and Zhang. This is an open-access article distributed under the terms of the Creative Commons Attribution License (CC BY). The use, distribution or reproduction in other forums is permitted, provided the original author(s) and the copyright owner(s) are credited and that the original publication in this journal is cited, in accordance with accepted academic practice. No use, distribution or reproduction is permitted which does not comply with these terms. 


\section{APPENDIX}

The cerebral cortex was parcellated into 68 functional areas, 34 for each hemisphere. The indexes and their corresponding names of function regions are provided in Appendix Table A1.

TABLE A1 | Cerebral cortex was parcellated into 68 functional areas, 34 for each hemisphere.

\begin{tabular}{|c|c|c|c|c|c|c|c|}
\hline Index & Region & Index & Region & Index & Region & Index & Region \\
\hline 1 & lh.bankssts & 18 & Ih.parahippocampal & 35 & rh.bankssts & 52 & rh.parahippocampal \\
\hline 2 & Ih.caudalanteriorcingulate & 19 & Ih.parsopercularis & 36 & rh.caudalanteriorcingulate & 53 & rh.parsopercularis \\
\hline 3 & Ih.caudalmiddlefrontal & 20 & Ih.parsorbitalis & 37 & rh.caudalmiddlefrontal & 54 & rh.parsorbitalis \\
\hline 4 & Ih.cuneus & 21 & Ih.parstriangularis & 38 & rh.cuneus & 55 & rh.parstriangularis \\
\hline 5 & Ih.entorhinal & 22 & Ih.pericalcarine & 39 & rh.entorhinal & 56 & rh.pericalcarine \\
\hline 6 & Ih.frontalpole & 23 & Ih.postcentral & 40 & rh.frontalpole & 57 & rh.postcentral \\
\hline 7 & Ih.fusiform & 24 & Ih.posteriorcingulate & 41 & rh.fusiform & 58 & rh.posteriorcingulate \\
\hline 8 & Ih.inferiorparietal & 25 & Ih. precentral & 42 & rh.inferiorparietal & 59 & rh.precentral \\
\hline 9 & Ih.inferiortemporal & 26 & Ih.precuneus & 43 & rh.inferiortemporal & 60 & rh.precuneus \\
\hline 10 & Ih.insula & 27 & Ih.rostralanteriorcingulate & 44 & rh.insula & 61 & rh.rostralanteriorcingulate \\
\hline 11 & Ih.isthmuscingulate & 28 & Ih.rostralmiddlefrontal & 45 & rh.isthmuscingulate & 62 & rh.rostralmiddlefrontal \\
\hline 12 & Ih.lateraloccipital & 29 & Ih.superiorfrontal & 46 & rh.lateraloccipital & 63 & rh.superiorfrontal \\
\hline 13 & Ih.lateralorbitofrontal & 30 & Ih.superiorparietal & 47 & rh.lateralorbitofrontal & 64 & rh.superiorparietal \\
\hline 14 & Ih.lingual & 31 & Ih.superiortemporal & 48 & rh.lingual & 65 & rh.superiortemporal \\
\hline 15 & Ih.medialorbitofrontal & 32 & Ih.supramarginal & 49 & rh.medialorbitofrontal & 66 & rh.supramarginal \\
\hline 16 & Ih.middletemporal & 33 & Ih.temporalpole & 50 & rh.middletemporal & 67 & rh.temporalpole \\
\hline 17 & Ih.paracentral & 34 & Ih.transversetemporal & 51 & rh.paracentral & 68 & rh.transversetemporal \\
\hline
\end{tabular}

The prefix of Ih indicates left hemisphere, and rh represents right hemisphere. The numbers are the indexes of function regions. 